\title{
The enhanced transcriptional activity of the V-val subtype of Epstein-Barr virus nuclear antigen 1 in epithelial cell lines
}

\author{
SHI-JUAN MAI, DAN XIE, YU-FAN HUANG, FENG-WEI WANG, YI-JI LIAO, \\ HAI-XIA DENG, WEN-JU LIU, WEN-FENG HUA and YI-XIN ZENG \\ Department of Experimental Research, State Key Laboratory of Oncology in Southern China, Cancer Center,
Sun Yat-sen University, 651 DongFeng Road East, Guangzhou 510060, P.R. China
}

Received November 9, 2009; Accepted December 22, 2009

DOI: $10.3892 /$ or_00000779

\begin{abstract}
The Epstein-Barr virus encoded nuclear antigen 1 (EBNA1) is required for the replication and maintenance of the episomal EBV genome and for the transactivation of viral gene expression. EBNA1 has been classified into five subtypes, among which the V-val subtype was reported to be associated with nasopharyngeal carcinoma (NPC). Here we report a higher transcriptional activity of the $\mathrm{V}$-val subtype of EBNA1 than for the prototype derived from B95.8 cells to transactivate FR-containing luciferase plasmid, which was mainly a consequence of the mutations in the carboxyterminus of EBNA1. This interpretation was further supported by the finding that the variant form of EBNA1 has a higher binding affinity for the FR sequence than the prototype by electrophoretic mobility shift assays. The functional advantage of the V-val EBNA1 investigated in this study may contribute to the oncogenesis of NPC.
\end{abstract}

\section{Introduction}

Nasopharyngeal carcinoma (NPC) is a human malignancy derived from epithelial cells that is rare in most parts of the world but is common in Southern China, Southeast Asian, and some North African countries. It has been convincingly shown to be an Epstein-Barr virus (EBV)-associated cancer via evidence that EBV is harbored in almost every undifferentiated NPC tumor irrespective of its geographic distribution. Despite the extremely variable incidence of NPC that is dependent on the geographic region, $\mathrm{EBV}$ is a ubiquitous human herpes virus that infects more than $90 \%$ of people worldwide such that many of these infected individuals

Correspondence to: Dr Yi-Xin Zeng, Sun Yat-sen University Cancer Center, 651 Dongfeng Road East, Guangzhou 510060, P.R. China

E-mail: zengyix@mail.sysu.edu.cn

Key words: EBV, EBNA1, sequence variation, transcriptional activation remain lifelong carriers of the virus without showing any overt or serious consequences of the disease. Thus, it has been hypothesized that specific EBV strains prevalent in NPChigh incidence areas may have higher transforming potentials that contribute to the development of NPC.

Infection in EBV-associated tumors is strictly latent, with only a limited range of viral genes being expressed by the cells. EBV encoded nuclear antigen 1 (EBNA1) is the only EBV protein consistently expressed in all infected proliferating cells, and it plays several important roles in EBV latent infection, including the initiation of EBV DNA replication, the mitotic segregation of the EBV genomes, and the transcriptional activation of other EBV latency proteins by binding directly to specific recognition sites in the latent replication origin region, oriP, of EBV DNA as a homodimer (1). EBV DNA replication initiates within the dyad symmetry (DS) element of oriP, which contains four EBNA1 binding sites $(2,3)$. The partitioning of the episomes involves EBNA1 binding to the family-of-repeat (FR) elements of oriP, which contains 20 EBNA1 recognition sites (4). FR is also a transcriptional enhancer through which EBNA1 supports the activity of the EBV latent membrane protein promoter and the $\mathrm{Cp}$ promoter (5-7). The EBNA1 protein also autoregulates the Qp promoter, which is the promoter for EBNA1 gene expression during latency I and II (8).

The predicted amino acid sequence of EBNA1, which consists of 641 amino acid residues, can be separated into unique amino-terminal (residues 1-89) and carboxy-terminal (residues 328-641) domains joined by an internal glycine/ alanine-rich short repeat (GAR) sequences (9). EBNA1 residues that mediate DNA binding and dimerization have been localized to the carboxy-terminal portion of the protein between amino acids 459 and 607. Amino acids 1-89 and 322-379 cooperatively mediate the association with cell chromosomes and are essential for episome persistence and transcription (10), whereas amino acids 379 through 386 form a nuclear localization sequence, and amino acids 605 through 641 comprise an acidic activating domain.

Based on the variation of the amino acid at residue 487 , EBNA1 was classified into two prototypes (P) and three variant (V) subtypes: P-ala (B95.8 prototype), P-thr (amino acid 487 ala to thr), V-val, V-leu and V-pro (11). A close relationship of V-val EBNA1 with NPC was suggested by its preference to infect NPC cells. V-val was the only subtype 
of EBNA-1 observed in a total of 20 cases of Chinese NPC biopsies in the study reported by Snudden et al (12) and Wang et al (13). In our previous study, $96.55 \%$ (84/87) of the NPC specimens contained the V-val subtype as the sole species of EBNA1, while multiple EBNA-1 subtypes, which included V-val, P-ala and/or V-thr EBNA1, were simultaneously presented in the peripheral blood lymphocytes of $77.08 \%(111 / 144)$ of EBV healthy carriers from the Guangzhou area in Southern China $(14,15)$. Therefore, it is reasonable to infer some additional risk for those individuals with V-val EBNA1 over the other subtypes in regard to the development of NPC. According to a comparison of the sequencing results of the $\mathrm{V}$-val and the prototype EBNA1 coding region, most of the variations of V-val EBNA1 would lead to substantial amino acid alterations in apparently functionally important regions, which may result in significant biological differences between the V-val and the prototype EBNA1.

In our previous study, we have investigated the functional difference between $\mathrm{V}$-val and the prototype EBNA1 in a human epithelial cell line. The coding regions of the P-ala and the $\mathrm{V}$-val subtypes of EBNA1 were cloned into the pGFP-C2 vector and transfected into 293 cells. The results of flow cytometry analyses indicated that the relative average intensity of fluorescence after 14 days of G418 selection in the V-val-EBNA1/293 cells was statistically higher than that observed for the P-ala-EBNA1/293 cells $(\mathrm{P}<0.05$, t-test), which indicated an enhanced maintenance and/or transactivation function of V-val EBNA1 over the prototype (16).

In order to confirm the functional advantage of $\mathrm{V}$-val EBNA1 and further locate the region responsible for this statistically significant difference, the EBNA1 mutants with their amino- and carboxy-terminal derived from the $\mathrm{V}$-val or from the prototype were constructed and cotransfected with an FR-containing luciferase reporter plasmid in the epithelial cell lines used in the study presented herein. The DNA binding affinity to the FR sequence was compared between the V-val and the prototype EBNA1 using an electrophoretic mobility shift assay. Our results indicate a stronger transcriptional activity of V-val EBNA1 compared with the prototype, which may be due to the higher DNA binding affinity to the FR sequence. Moreover, this functional advantage may contribute to the oncogenesis of NPC.

\section{Materials and methods}

Cell lines. The spontaneously transformed human keratinocyte cell line $\mathrm{HaCaT}$ that was derived from normal human trunk skin was used in this study. The poorly differentiated NPC cell line CNE-2 and human embryonic kidney epithelial cell line 293, were used to confirm the results of the reporter assays. All cells were maintained in RPMI-1640 medium containing $10 \%$ fetal bovine serum and incubated at $37^{\circ} \mathrm{C}$ in an incubator with $5 \% \mathrm{CO}_{2}$. The NPC biopsies were collected from the Cancer Center at Sun Yat-sen University.

Construction of mutants of EBNA1 lacking amino acids 91 to 321. In order to compare the expression and activity of the prototype and the mutated EBNA1 proteins, a series of pcDNA3. $1^{+}$vectors were created using the same expression cassette that encodes different EBNA1 genes. The coding region of the truncated forms of EBNA1 containing a deletion of most of the Gly-Ala repeat region (amino acids 91 through 321) was included, since a previous study showed that this region can be removed with little or no effect on the function of EBNA1 (17). The EBNA1 derivatives expressed from these plasmids were predicted to have a molecular weight of $43 \mathrm{kDa}$.

The prototype and the V-val subtype of EBNA1 were amplified using template DNA extracted from the B95.8 cell line and the NPC biopsy. The amino-terminal (amino acids 1-90) region of the EBNA1 gene was amplified by two rounds of polymerase chain reaction (PCR) amplification using primers that introduced a 6-His tag, a Kozark sequence and the BamHI and HindIII restriction sites (sense primer for first round of PCR: 5'-GAACACCACCATCACCATCACA TGTCTGACG-3', antisense primer for first and second round of PCR: 5'-ACTAGTAAGATTTCCTGTTCCACCGTGG-3' and a sense primer for second round of PCR: 5'-ATACTC GAGGGATCCACCATGGAACACCAC-3'). The carboxyterminus (amino acids 322-641) of EBNA1 was amplified using primers with HindIII and EcoRI restriction sites (sense primer: 5'-ACTAGTAAGCTTGCAGGAGCAGGAGGT GGA-3' and an antisense primer: 5'-TACTCGAGGAATT CTCACTCCTGCCCTT-3'). Pyrobest DNA polymerase (Takara Biotechnology, Dalian, China) with proof-reading activity was used for the amplification step. The prototype and variant amino- and carboxy-terminal region of the EBNA1 fragments were purified from agarose gels, digested with HindIII, and subsequently ligated. In order to test the different effects of the amino- and carboxy-terminal variations on the transcriptional activity of EBNA1, the fragments derived from the prototype and the V-val subtype were ligated. PCR was performed using the ligation mixture as a template with the EBNA1 amino- and carboxy-terminal primers. PCR products were gel purified, digested with $B a m \mathrm{HI}$ and EcoRI, and ligated into the BamHI and EcoRI sites of pcDNA3.1 . The resulting constructs expressed EBNA1 mutants as aminoand carboxy-terminal fusion proteins that lacked the GlyAla repeat region (amino acids 91-321) and instead were replaced with the 6 nucleotide sequence AAGCTT (HindIII restriction site). Four EBNA1 mutants were subsequently constructed and included pp-EBNA1 (derived from B95.8 cells, p, prototype), vv-EBNA1 (derived from NPC, v, variant), pv-EBNA1 (amino-terminus derived from B95.8 and carboxy-terminus derived from NPC), and vp-EBNA1 (amino-terminus derived from NPC and carboxy-terminus derived from B95.8). These desired constructs were verified by sequencing analysis and further amplified and purified using QIAgen maxi prep protocols.

Construction of reporter gene plasmids. The FR element of ori $\mathrm{P}$ (EBV coordinates 7421-8042) was amplified by PCR using a sense primer (5'-TGTGGATCCGGGTATCATATG CTGAC-3'), an antisense primer (5'-CACGGATCCTAAT GTTGCCATGGGTA-3'), and DNA extracted from B95.8 cells as a template. The resulting fragment contained two engineered BamHI sites at positions 7421 and 8042. The FR fragment was then digested with BamHI and was used for ligation into the site of the pGL3-SV40 promoter vector. In 
the resulting construct, referred to here as pSV40-luciferaseFR, the FR element is positioned 510 bp downstream from the luciferase reporter gene. Recombinants were confirmed by enzyme digestion and sequencing analyses.

EBV Cp (-121 to +98 relative to the Cp start site, coordinates 11215-11434 in the B95-8 genome) and LMP1TR (-123 to +221 relative to the LMP1-TR promoter start site, coordinates 169976-170319 in the B95-8 genome) promoter sequences were amplified from B95-8 DNA by PCR and used to substitute the simian virus 40 (SV40) promoter in the pSV40-luciferase-FR plasmid. The pCpFR and pLMP1-TR-FR constructs were confirmed by sequencing.

Western blot analysis. HaCaT cells in 100-mm dishes were transfected with $10 \mu \mathrm{g}$ of pcDNA3.1+ vector, pc3-ppEBNA1, pc3-pv-EBNA1, pc3-vp-EBNA1 and pc3-vvEBNA1 plasmids, respectively. The culture medium was removed $48 \mathrm{~h}$ post-transfection, and the cells were washed once in PBS. The cells in each dish were then lysed in M-PER Mammalian Protein Extraction Reagent (Pierce, Rockford, IL) with $1 \mathrm{mM}$ phenylmethylsulfonyl fluoride (PMSF) and a Protease Inhibitor Cocktail (Sigma). The cell debris was removed by centrifugation at $14,000 \mathrm{~g}$ for $5 \mathrm{~min}$. The protein concentration in the supernatant was determined using the BCA protein assay kit (Pierce). The cellular protein was used for the Western blot analysis and electrophoretic mobility shift assay. For the Western blot assay, the lysate supernatant equivalent of $40 \mu \mathrm{g}$ of protein was separated by electrophoresis on a $10 \%$ SDS-polyacrylamide gel. The proteins were then transferred to a polyvinylidene fluoride membrane (Roche). After blocking, the membranes were incubated with anti-His tag Mouse Monoclonal Antibody (Novagen, Madison, WI), followed by an HRP-conjugated secondary antibody. The proteins were detected by incubation with LumiGLO ${ }^{\mathrm{TM}}$ (Cell Signaling Technology, Beverly, MA) and captured on Kodak X-ray film.

Reporter assays. HaCat cells were plated in 24-well plates at a density of $5 \times 10^{4}$ cells/well and reached $70-80 \%$ confluence $24 \mathrm{~h}$ later. Subsequently, $0.4 \mu \mathrm{g}$ of pcDNA3.1+ plasmid expressing pp-EBNA1, pv-EBNA1, vp-EBNA1, vv-EBNA1, or no EBNA1 was combined with $0.1 \mu \mathrm{g}$ of the pSV40luciferase-FR reporter construct and $10 \mathrm{ng}$ of pRL-TK (Promega, Madison, WI) in $1 \mu \mathrm{l}$ of the FuGENE 6 reagent diluted in $10 \mu \mathrm{l}$ of serum-free medium. The experiments were performed in three replicate wells for each transfection. The transfection reagent: DNA complex was incubated for $15 \mathrm{~min}$ at room temperature and then was added dropwise to cells. After incubation for $12-16 \mathrm{~h}$ at $37^{\circ} \mathrm{C}$, the cells were given fresh medium. After $48 \mathrm{~h}$ of incubation at $37^{\circ} \mathrm{C}$, the dual-luciferase reporter assay was performed using the luciferase assay system (Promega) according to the manufacturer's instructions. The cells were washed with $1 \mathrm{X}$ PBS and lysed with $100 \mu 1$ 1X PLB for each well. Subsequently, $20 \mu 1$ of the cell lysate was mixed with $50 \mu 1$ of LARII, and the luminescence was immediately measured using a Sirius Luminometer V3.1 (Berthold Detection Systems, Germany) that recorded the firefly luciferase activity measurements (F-value). The sample was then removed from the luminometer and added to $50 \mu 1$ of the $1 \mathrm{X}$ Stop\&Glo reagent, and the Renilla luciferase activity measurement (R-value) was recorded again. The relative light units (RLU) were calculated by dividing the R-value by the F-value and represented the transcriptional activity with the normalized transfection efficiency of each group. The reporter assay results are presented as an increase ( $n$-fold) in the level of transcription. The increase in transcription was calculated by dividing the RLU obtained from cells transfected with the various derivatives of EBNA1 by the RLU obtained from cells transfected with the empty vector. The experiment was performed independently at least three times.

The experiment was performed by repeating the same procedure as above to confirm the result of reporter assay using the CNE-2 and 293 cell lines. The EBV-negative NPC cell line (CNE-2) was chosen because of its lack of endogenous EBNA1 expression. Prior to using the luciferase assay, either pp- or vv-EBNA1 was cotransfected with pSV40luciferase that lacked the inserted FR element in HaCat cells in order to confirm the necessity of the FR element in EBNA1-mediated transactivation.

The EBNA1-mediated transcriptional activation of the EBV promoter was measured by replacing the SV40 promoter in the pGL3-FR plasmid with Cp or LMP1-TR. Fixed amounts $(0.4 \mu \mathrm{g})$ of the EBNA1 derivatives were cotransfected with $0.1 \mu \mathrm{g}$ of $\mathrm{pCp}-\mathrm{FR}$ or pLMP1-TR-FR into HaCat cells, and the luciferase activity was measured $48 \mathrm{~h}$ after cotransfection. For each group, the experiments were performed in 3 replicate wells.

To evaluate the dynamic changes in luciferase activity during 4-day culture, the HaCat cells were plated in 24-well plates at a low density of $1.5 \times 10^{4}$ cells/well and transfected with the same procedure as described above $24 \mathrm{~h}$ after the cells reached $30-40 \%$ confluence. These experiments were performed in 12 replicate wells for each transfection. Specifically, $0.4 \mu \mathrm{g}$ of pcDNA3 $31^{+}$plasmid expressing ppEBNA1, pv-EBNA1, vp-EBNA1, vv-EBNA1, or no EBNA1 was combined with $0.1 \mu \mathrm{g}$ of the pSV40-luciferase-FR reporter construct and $10 \mathrm{ng}$ of pRL-TK in $1 \mu \mathrm{l}$ of the FuGENE 6 reagent. In addition to the empty vector, a mock control with a non-sense mutation in the beginning of the pp-EBNA1 sequence was also included. These cells were grown for 4 days, collected, and screened for the presence of luciferase activity at 24, 48, 72, and 96 h post-transfection.

Electrophoretic mobility shift assay. Seven of the amino acid mutations identified in V-val EBNA1 occur within the DNA binding and dimerization domain. In order to determine whether the changes have an effect on the binding affinity, we performed electrophoretic mobility shift assays (EMSA) to compare the ability of the prototype and V-val EBNA1 to bind DNA. The biotin end-labeled single-stranded oligonucleotide (5'-Biotin-TAGCATATGCTACCCGGAT ACAGATTAGGATAGCATATACTACCCAGATATAGAT TAGGA-3') and its complementary oligomer (3'-ATCG TATACGATGGGCCTATGTCTAATCCTATCGTATATG ATGGGTCTATATCTAATCCT-Biotin-5'), which corresponded to the EBNA1 binding site in the FR element, were synthesized (Shanghai Invitrogen Biotech Co., Ltd., Shanghai, China) and subjected to double-stranded annealing. The 
whole cellular proteins from the pp-EBNA1 or vv-EBNA1 transfected HaCat cells were extracted as described above. EMSA was performed using the LightShift ${ }^{\circledR}$ Chemiluminescent EMSA Kit according to the manufacturer's protocol (Pierce). The binding reaction was carried out in a total volume of $10 \mu \mathrm{l}$ containing $3 \mu \mathrm{g}$ of cellular extract, $10 \mathrm{fmol}$ of biotin-labeled oligonucleotides and $1 \mathrm{X}$ binding buffer comprising $10 \mathrm{mM}$ Tris, $50 \mathrm{mM} \mathrm{KCl,} 1 \mathrm{mM}$ DTT, $5 \mathrm{mM}$ $\mathrm{MgCl}_{2}, 2.5 \%$ glycerol, $0.05 \% \mathrm{NP}-40$ and $0.5 \mu \mathrm{g}$ poly $(\mathrm{dI} \cdot \mathrm{dC})$. The protein/DNA complexes were incubated for $20 \mathrm{~min}$ at room temperature and then subjected to vertical electrophoresis in a $6 \%$ Tris-borate-EDTA polyacrylamide gel. The gel was electrophoresed at $100 \mathrm{~V}$ in $0.5 \mathrm{X}$ Tris-borateEDTA buffer until the bromophenol blue dye had migrated approximately $2 / 3$ to $3 / 4$ of the length of the gel. Transfer was performed at $380 \mathrm{~mA}$ dor $30 \mathrm{~min}$. The transferred DNA was cross-linked to membrane and detected by chemiluminescence and then exposed to X-ray film.

Supershift experiments were carried out as described above except that the samples were preincubated for $1 \mathrm{~h}$ at $4^{\circ} \mathrm{C}$ in the presence of $1 \mu \mathrm{g}$ of anti-His tag antibody (Novagen). For the competition binding assay, unlabeled double-stranded oligonucleotides were used at a 50-fold excess of biotin-labeled probe.

Statistics. Data are presented as the mean \pm SD. The differences were assessed by analysis of variance with double-sided t-test for comparisons within multiple groups. Statistical significance was defined as $\mathrm{P}<0.05$.

\section{Results}

The expression of EBNAl derivatives in the HaCat cell line. The comparative expression level and molecular weight of the EBNA1 derivatives were analyzed in the HaCat transformants via Western analysis using the anti-His tag antibody. The total protein fraction was prepared and equal amounts of this fraction were separated using SDS-polyacrylamide gel electrophoresis. As illustrated in Fig. 1, the EBNA1 proteins from the four derivatives pp-EBNA1, pv-EBNA1, vp-EBNA1 and vv-EBNA1 were detectable at equivalent levels (Fig. 1, lanes 3-6). In this set of experiments, the untransfected HaCat cells (Fig. 1, lane 1) and cells transfected with pcDNA3.1+ (Fig. 1, lane 2) were used as controls. The molecular weights of the recombinant EBNA1 proteins were predicted to be $43 \mathrm{kDa}$.

The transactivation of the FR-containing luciferase gene is higher in $v v$-EBNAl-expressing cells. The different derivatives of EBNA1 and the empty vector pcDNA3.1+ were co-transfected with pSV40-luciferase-FR and the internal control pRL-TK into cells. The relative light units (RLU) were calculated using the F/R value obtained after quantification of the luciferase activity in the transfected cells. We analyzed four derivatives of EBNA1 in order to compare the different contribution of the fragments to the transcription of the FR-plasmid.

The mean luciferase results that were corrected for the transfection efficiency obtained from at least three independent transfections performed in duplicate were depicted graphically (Fig. 2A). The increases in the transcription level

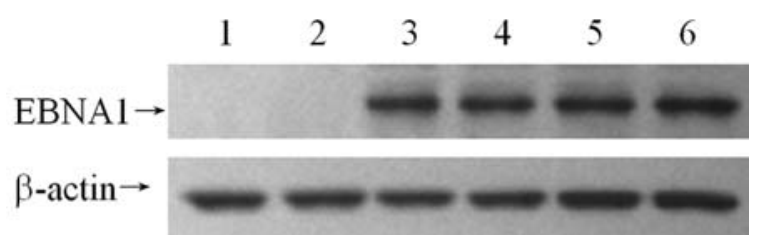

Figure 1. Western blot assays demonstrating the expression of the various derivatives. Forty micrograms of protein extracted from the transfected HaCat cells were resolved by $10 \%$ SDS-polyacrylamide gels and probed with the anti-His tag primary antibody (Upstate). The antibody did not detect a signal in untransfected cells (lane 1) or in cells transfected with the empty vector (lane 2). Western blot analysis shows that the expression of pp-EBNA1 (lane 3), pv-EBNA1 (lane 4), vp-EBNA1 (lane 5) and vvEBNA1 (lane 6) with a putative molecular weight of $43 \mathrm{kDa}$. Notably, $\beta$-actin was used as the internal control.

over that of the empty vector mediated by pp-EBNA1 [mean increase ( $\mathrm{n}$-fold) over the empty vector, $10.78(\mathrm{P}=0.0004)]$, pv-EBNA1 [mean increase ( $\mathrm{n}$-fold) over the empty vector, $14.16\left(\mathrm{P}=6.99 \times 10^{-5}\right)$ ], vp-EBNA1 [mean increase ( $\mathrm{n}$-fold) over the empty vector, $10.98(\mathrm{P}=0.0004)]$, and vv-EBNA1 [mean increase ( $\mathrm{n}$-fold) over the empty vector, 14.57 $\left.\left(\mathrm{P}=2.28 \times 10^{-5}\right)\right]$ are statistically significant. The apparent increases in transcription over the prototype-type EBNA-1 as mediated by the pv-EBNA1 and vv-EBNA1 derivatives were statistically significant $(\mathrm{P}<0.05)$. However, the derivatives of pp-EBNA1 and vp-EBNA1 were found to show no statistically significant difference between their levels of transcription $(\mathrm{P}>0.05)$. These results suggest that variations in the carboxy-terminal region of V-val EBNA1 enhanced the transactivation activity.

The experiment was repeated by using the CNE-2 (Fig. 2B) and 293 (Fig. 2C) cell lines using the same procedure. Interestingly, similar results were observed for these two cell lines. When cotransfected with the pSV40-luciferase without the FR in HaCat cells, the transactivating activity of ppEBNA1 and vv-EBNA1 was found to be equivalent to that of the empty vector, demonstrating the requirement of the FR element in EBNA1-mediated transactivation (Fig. 2D). As the SV40 promoter in luciferase reporter was replaced by EBV promoter Cp or LMP1-TR, similar results were obtained from the reporter assay. The vv-EBNA1-mediated transcriptional activities of pCp-FR and pLMP1-TR-FR were 2.56 and 2.15 times higher than the corresponding pp-EBNA1-mediated transcriptional activities, respectively (Fig. 2E and 2F). The differences were statistically significant $(\mathrm{P}<0.05$ for both differences, $t$-test $)$.

The luciferase activity was measured at 24, 48, 72, and $96 \mathrm{~h}$ after transfection of the EBNA1 derivatives. The RLU detected in the empty pcDNA3.1+ vector transfected cells was very low and decreased from $0.56 \pm 0.05$ to $0.11 \pm 0.01$ over a period of 3 days, while the RLU detected in EBNA1 transfected cells increased during the same period of time (from $2.34 \pm 0.36$ to $5.98 \pm 1.25$ for pp-EBNA1, from $3.52 \pm 0.56$ to $7.83 \pm 0.71$ for pv-EBNA1, from $4.00 \pm 0.48$ to $6.16 \pm 0.49$ for vp-EBNA1, from $4.81 \pm 0.42$ to $12.61 \pm 0.31$ for vv-EBNA1). Interestingly, only low levels of luciferase activity could be detected in the mock group over this period (from $0.56 \pm 0.19$ to $0.90 \pm 0.21$ ). The increase in transcription calculated by dividing the RLU obtained from cells trans- 
A

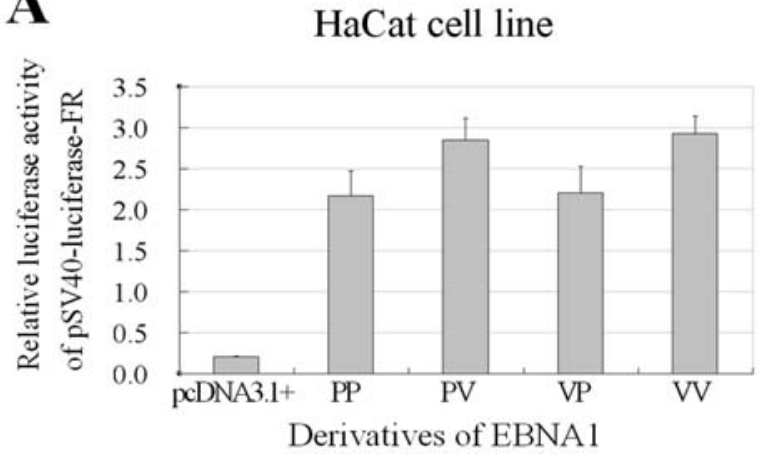

C

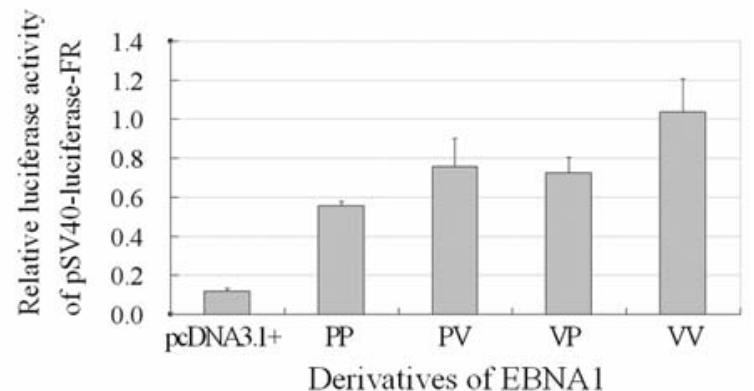

E

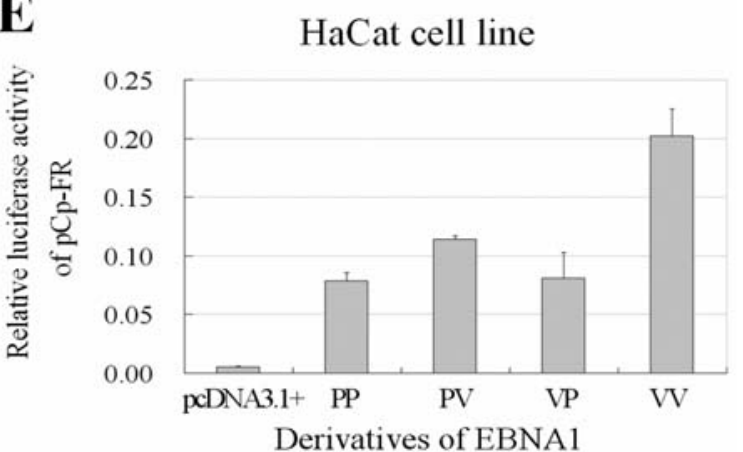

B

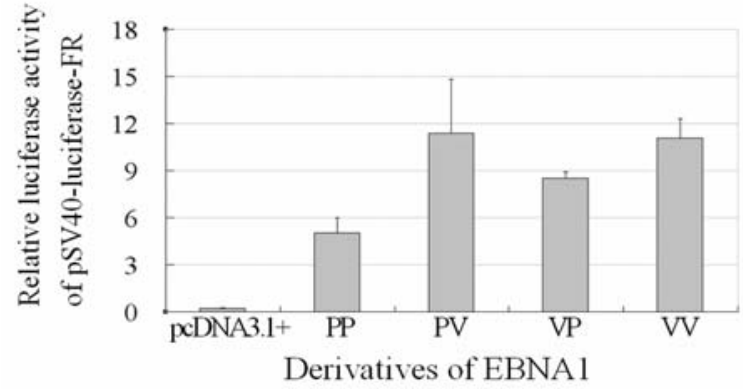

D

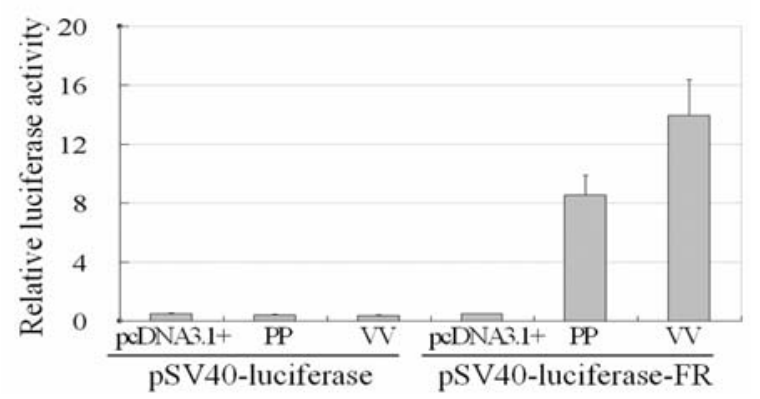

F

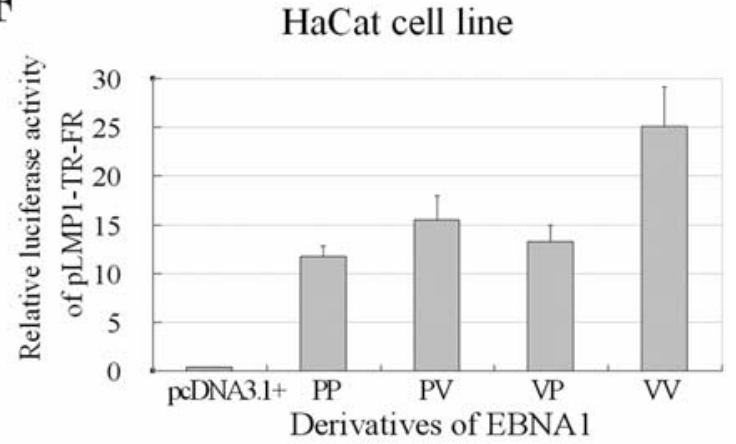

Figure 2. Derivatives of EBNA1 support transcription through the FR to different degrees in epithelial cell lines. (A) Plasmids expressing the indicated EBNA1 derivatives or the empty vector were cotransfected with pSV40-luciferase-FR in HaCat cells and the luciferase activity was subsequently measured $48 \mathrm{~h}$ later. (B and C) The reporter assay was repeated in CNE-2 (B) and 293 (C) cell lines following the exact same procedure. In the two aforementioned cell lines, the transactivating activity of vv-EBNA1 and pv-EBNA1 was significantly higher than that observed for pp-EBNA1 (P<0.05, $t$-test), and pv-EBNA1 was consistently more active than vp-EBNA1. (D) A cotransfection of $0.4 \mu \mathrm{g}$ of pcDNA3.1 ${ }^{+}$, pc3-pp-EBNA1, or pc3-vv-EBNA1 with $0.1 \mu \mathrm{g}$ of the pSV40luciferase-FR reporter or pSV40-luciferase vector without insertion of the FR element was performed. The luciferase reporter assay was then carried out $48 \mathrm{~h}$ post-transfection using the luciferase assay system according to the manufacturer's instructions. Neither pp-EBNA1 nor vv-EBNA1 promoted considerable transcription of the pSV40-luciferase plasmid. (E and F) Plasmids expressing the indicated EBNA1 derivatives or the empty vector were cotransfected with Cp (E)- or LMP1-TR (F)-derived FR-containing luciferase reporter plasmid in HaCat cells by using the procedure described above. The results above were normalized for Renilla luciferase derived of the plasmid expressing Renilla luciferase. The mean \pm SD of three independent experiments was shown.

fected with the various derivatives of EBNA1 by the RLU obtained from cells transfected with the empty vector is shown in Fig. 3. The luciferase activity for the vv-EBNA1/ HaCat cells was consistently higher than that observed for the pp-EBNA1 over the three-day period.

$v v$-EBNAl protein binds the FR sequence with higher affinity. The DNA binding abilities of the prototype EBNA1 and the V-val EBNA1 were compared via the EMSA by using a synthetic 60 -mer oligonucleotide containing the FR binding site and the cellular protein extracted from the pp-EBNA1 and vv-EBNA1 transfected HaCat cell lines as the substrate
(Fig. 4). Notably, the addition of an excess of cold probe inhibited the DNA-protein complex formation ability. Moreover, the addition of the anti-His tag antibody effectively inhibited the interaction between the cellular protein and the biotin-labeled FR probe as evidenced by a dramatic shift of the band. The EMSA bands were scanned and quantified densitometrically using the Bio-Rad Quantity One software. In 3 separate experiments, the intensities of the FR-binding band with vv-EBNA1 were 1.25, 1.39, and 1.5 times stronger than the intensities of the corresponding bands for pp-EBNA1. Although the difference was modest, it was consistent in the replicated assays, thereby indicating that the DNA-binding 


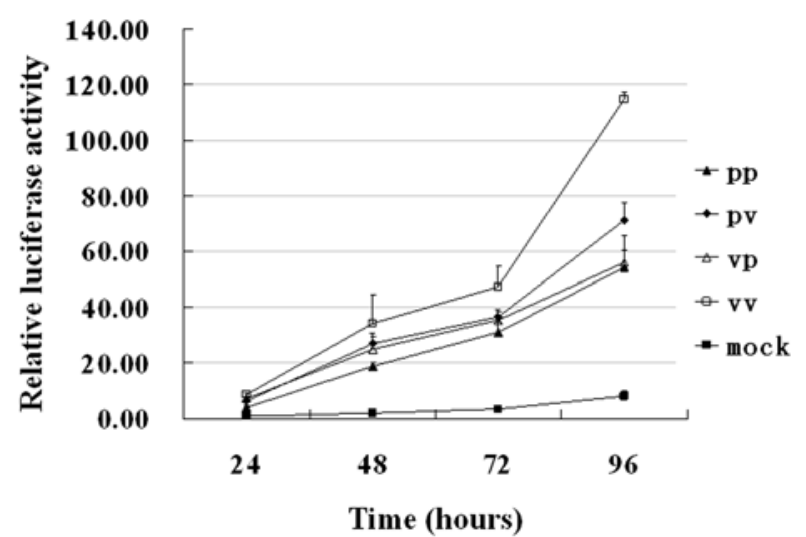

Figure 3. Dynamic changes in the luciferase activity of EBNA1-expressing cells during a four-day culture period. HaCat cells were cotransfected with the pSV40-luciferase-FR reporter plasmid and with pcDNA3.1 ${ }^{+}$plasmids expressing no EBNA1 (empty vector), pp-EBNA1 (solid triangles), pvEBNA1 (solid diamonds), vp-EBNA1 (open triangles), vv-EBNA1 (open squares) or a mock control (solid squares) that contained a non-sense mutation in the beginning of the pp-EBNA1 sequence. Equal amounts of lysate were prepared $24,48,72$, and $96 \mathrm{~h}$ post-transfection and were used for the measurement of the luciferase activity. The value of the relative luciferase activity indicated in the ordinate showed the increased transcription level as mediated by the EBNA1 derivatives over that of the empty vector. The error bars indicated the standard error of the mean for each group.

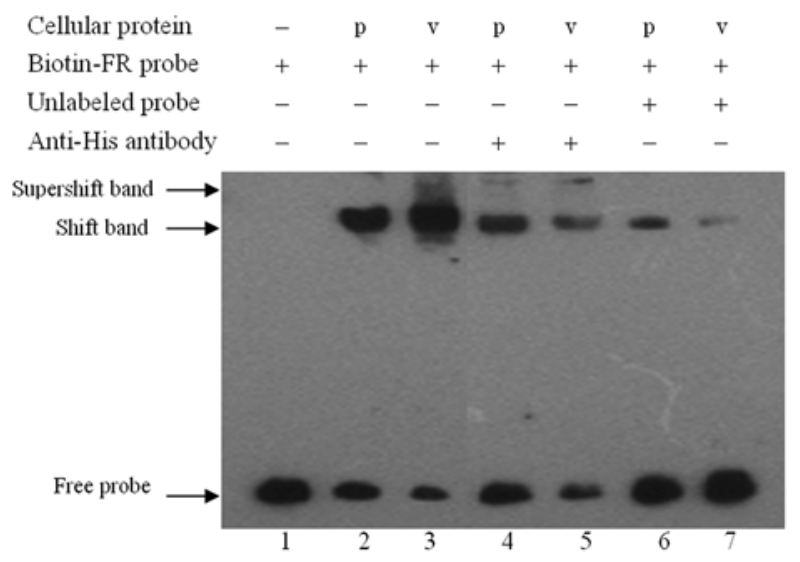

Figure 4. The higher DNA binding affinity of vv-EBNA1 to the FR sequence upon comparison to that of pp-EBNA1. EMSA experiments were performed using the total cellular extract of HaCat cells after transfection of pp-EBNA1 or vv-EBNA1 and the biotin labeled FR probes, which were used as substrate bait. The DNA-protein complex formation was shown in lanes 2 and 3, and the complex was competed out (with cold oligonucleotides) by a 50-fold molar excess of unlabeled oligonucleotide (lanes 6 and 7). The supershifted band was observed with antibodies against the His tag (lanes 4 and 5). The panel shows a representative autoradiograph from three independent experiments. The band intensity of pp-EBNA1 (lane 2) and vv-EBNA1 (lane 3) was determined using densitometric scanning and the ratio of vv-EBNA1/pp-EBNA1 was calculated to be 1.5 .

affinity of vv-EBNA1 was higher than that of pp-EBNA1. The representative result is shown in Fig. 4.

\section{Discussion}

Interestingly, while EBV infects most of the global population, only a small percentage of infected individuals develop EBV-associated malignancies, one of which is known as
NPC. NPC occurs at a high frequency in Asian populations, especially among people of Cantonese ancestry. We identified a unique EBV strain GD1 (GenBank accession no. AY961628) that notably differs from the B95.8 prototype and is widespread in Guangdong (18). The contribution of the GD1 substrain of EBV to NPC development is therefore certainly worthy of further investigation.

The V-val subtype of the EBNA1 strain is identical to the EBV strain GD1 and has been determined to be closely associated with the incidence of NPC in the Asian region, which suggests a functional advantage of V-val EBNA1 over the other subtypes regarding the oncogenesis of NPC. According to our previous study, a higher transactivation ability was observed for V-val EBNA1 when compared to the prototype by using GFP as an indicator (16), which was a result later supported by a study published by Do et al (19).

In order to evaluate the contribution of the EBNA1 fragments to the enhanced transactivation function, constructs containing a variety of amino- and carboxy-terminal EBNA1 fragments were examined herein for their ability to transactivate the FR containing luciferase reporter plasmid epithelial cell lines. The transcription of the luciferase gene can be promoted by all four EBNA1 derivatives by a factor greater than 10-fold over that observed for the empty vector. The increases in the level of transcription as mediated by vv-EBNA1 and pv-EBNA1 were significantly higher than that observed for pp-EBNA1, which supports the hypothesis that V-val EBNA1 is more likely to be responsible for the enhanced transactivation ability than the prototype. We suggest that the enhanced transactivation ability of V-valEBNA1 was mainly contributed by the carboxy-terminus of EBNA-1 due to the observation that the luciferase activity was consistently higher in the pv-EBNA1-expressing cells than that observed for the vp-EBNA1-expressing cells in separate experiments. This conclusion is reasonable since the sequences that are essential for the EBNA1 transcriptional activation function were mainly located in the carboxyterminus. Specifically, EBNA1 amino acid residues 459-607 are responsible for the DNA binding and dimerization abilities, and the structural basis for DNA recognition and dimerization has been revealed through X-ray crystallography studies (20). It was reported that the deletion of amino acid residues 325 through 376 of EBNA1 severely affects the transactivation activity, indicating a major transcriptional role for this Gly-Arg-rich region $(21,22)$. Interestingly, 7 and 1 amino acid mutations were detected in amino acid residues 459 through 607 and 325 through 376 of V-val EBNA1, respectively. Furthermore, the dynamic changes in luciferase activity were detected over four consecutive days after the transfection of the EBNA1 mutants into $\mathrm{HaCaT}$ cells, where a significant and continued increase in reporter activity was observed. This increase in the level of expression may be due to the persistent ability of the plasmid to express the EBNA1 coupled with the selection of EBNA1-expressing cells in the culture, which would lead to the contribution of EBNA1 to the overall cell growth.

EBNA1 exerts its functions by binding to FR and DS in the origin of the viral plasmid replication (oriP) in the EBV genome. EBNA1 binds to sites in the DS in order to support 
the stable replication of oriP. The FR component has been shown to be responsible for efficient segregation, mitoticchromosome attachment of the DNA constructs, and the transcriptional activation ability of EBNA1 $(23,24)$. The binding ability to the DS domain has been examined by gel shift assays that indicated no major differences between P-ala and V-val (12). Thus, we determined the binding affinity of EBNA1 to the FR element that is known to contribute to plasmid maintenance and the overall transcriptional activation ability. The mechanism of the enhanced transactivation ability of V-val EBNA1 as indicated by the luciferase reporter assays performed herein was revealed by the EMSA which demonstrated that vv-EBNA1 has a higher affinity for the FR sequence than pp-EBNA1. The increased binding affinity of V-val EBNA1 to the FR would also improve its ability to maintain the plasmid, which may contribute to the outgrowth of the V-val viral substrain in NPC cells and lead to stable latent infection of EBV in nasopharyngeal mucosa.

EBNA1 activates the transcription of EBV Cp and the LMP1 promoter. As shown in our results, in the presence of the FR enhancer, the variant-EBNA1-mediated transactivations of $\mathrm{Cp}$ and the LMP1-TR promoter were significantly higher than those mediated by the prototype EBNA1. Although $\mathrm{Cp}$ is hypermethylated and silenced in NPCs (25), V-val EBNA1 might increase the Cp transcription in normal PBMCs and contribute to the persistence of latent EBV infection. The transactivation of the LMP1 promoter by EBNA1 might contribute to the transformation of host cells. In addition to the modulation of the EBV latent promoters (26), multiple reports have shown a correlation between EBNA1 expression and the activation of prominent cellular targets such as the anti-apoptotic bcl-2 family members, the $\mathrm{V}(\mathrm{D}) \mathrm{J}$ recombinase-activating genes RAG1 and RAG2, in addition to CD25 (27-29). These proteins may contribute to genetic instability and cellular transformation. Gene profiling analysis of EBNA1-expressing carcinoma cells revealed that EBNA1 can influence the expression of a range of cellular genes including those involved in translation, transcription, and cell signaling, resulting in effects that may contribute to the development of EBV-associated tumors (30). These reports indicate that the transactivation ability of EBNA1 may have important functional significance in the pathogenesis of EBV associated tumors.

It is likely that EBNA1 contributes to cell transformation by guaranteeing extrachromosomal viral maintenance in order to establish the latent status of EBV and foster the transcription of viral latent genes in addition to a bevy of cellular genes. We present here evidence to suggest the functional advantage of V-val EBNA1 over the prototype, may be helpful in the elucidation of the possible oncogenic potential of EBNA1.

\section{Acknowledgements}

This work is supported by grants from the National High Technology Research and Development Program of China (863 Program) (No. 2006AA02A404) and the Major State Basic Research Program of China (No. 2006CB910104).

\section{References}

1. Kieff E and Rickinson AB: Epstein-Barr virus and its replication In: Fields Virology. 4th edition. Knipe DM and Howley PM (ed.) Lippincott Williams and Wilkins Publications Corp., Philadelphia, pp2511-2574, 2001.

2. Gahn TA and Schildkraut CL: The Epstein-Barr virus origin of plasmid replication, oriP, contains both the initiation and termination sites of DNA replication. Cell 58: 527-535, 1989.

3. Niller HH, Glaser G, Knüchel R and Wolf H: Nucleoprotein complexes and DNA 5'-ends at oriP of Epstein-Barr virus. J Biol Chem 270: 12864-12868, 1995.

4. Lupton S and Levine AJ: Mapping of genetic elements of Epstein-Barr virus that facilitate extrachromosomal persistence of Epstein-Barr virus-derived plasmids in human cells. Mol Cell Biol 5: 2533-2542, 1985.

5. Gahn TA and Sugden B: An EBNA1-dependent enhancer acts from a distance of 10 kilobase pairs to increase expression of the Epstein-Barr virus LMP gene. J Virol 69: 2633-2636, 1995.

6. Puglielli MT, Desai N and Speck SH: Regulation of EBNA gene transcription in lymphoblastoid cell lines: characterization of sequences downstream of BCR2 (Cp). J Virol 71: 120-128, 1997.

7. Sugden B and Warren N: A promoter of Epstein-Barr virus that can function during latent infection can be transactivated by EBNA-1, a viral protein required for viral DNA replication during latent infection. J Virol 63: 2644-2649, 1989.

8. Nonkwelo C, Skinner J, Bell A, Rickinson A and Sample J: Transcription start sites downstream of the Epstein-Barr virus (EBV) Fp promoter in early-passage Burkitt lymphoma cells define a fourth promoter for expression of the EBV EBNA-1 protein. J Virol 70: 623-627, 1996.

9. Baer R, Bankier AT, Biggin MD, Deininger P, Farrell PJ, Gibson T, Hudson G, Satchwell S, Seguin C, Tuffnell P and Barrel B: DNA sequence and expression of the B95-8 EpsteinBarr virus genome. Nature 310: 207-211, 1984.

10. Hung SC, Kang MS and Kieff E: Maintenance of Epstein-Barr virus (EBV) oriP-based episomes requires EBV-encoded nuclear antigen-1 chromosome-binding domains, which can be replaced by high-mobility group-I or histone H1. Proc Natl Acad Sci USA 98: 1865-1870, 2001.

11. Bhatia K, Raj A, Guitierrez MI, Judde JG, Spangler G, Venkatesh $\mathrm{H}$ and Magrath IT: Variation in the sequence of Epstein Barr virus nuclear antigen 1 in normal peripheral blood lymphocytes and in Burkitt's lymphomas. Oncogene 13: 177-181, 1996.

12. Snudden DK, Smith PR, Lai D, Ng MH and Griffin BE: Alterations in the structure of the EBV nuclear antigen, EBNA1, in epithelial cell tumours. Oncogene 10: 1545-1552, 1995.

13. Wang WY, Chien YC, Jan JS, Chueh CM and Lin JC: Consistent sequence variation of Epstein-Barr virus nuclear antigen 1 in primary tumor and peripheral blood cells of patients with nasopharyngeal carcinoma. Clin Cancer Res 8: 2586-2590, 2002.

14. Mai SJ, Zhang XS, Li DJ, Shen GP, Jiang JH, Zhang RH, Yu XJ, Chen SP and Zeng YX: The character of EBNA1 gene variation in Cantonese and its association with nasopharyngeal carcinoma. Chin Sci Bull 49: 2640-2643, 2004.

15. Zhang XS, Wang HH, Hu LF, Li A, Zhang RH, Mai HQ, Xia JC, Chen LZ and Zeng YX: V-val subtype of Epstein-Barr virus nuclear antigen 1 preferentially exists in biopsies of nasopharyngeal carcinoma. Cancer Lett 211: 11-18, 2004.

16. Mai SJ, Ooka T, Li DJ, Shen GP, Jiang JH, Zhang RH, Yu XJ, Chen SP and Zeng YX: Functional advantage of NPC-related V-val subtype of Epstein-Barr virus nuclear antigen 1 compared with prototype in epithelial cell line. Oncol Rep 17: 141-146, 2007.

17. Yates JL and Camiolo SM: Dissection of DNA replication and enhancer activation functions of Epstein-Barr virus nuclear antigen 1. Cancer Cells 6: 197-205, 1988.

18. Zeng MS, Li DJ, Liu QL, Song LB, Li MZ, Zhang RH, Yu XJ, Wang HM, Ernberg I and Zeng YX: Genomic sequence analysis of Epstein-Barr virus strain GD1 from a nasopharyngeal carcinoma patient. J Virol 79: 15323-15330, 2005.

19. Do NV, Ingemar E, Phi PT, Jenny A, Chinh TT, Zeng Y and Hu L: A major EBNA1 variant from Asian EBV isolates shows enhanced transcriptional activity compared to prototype B95.8. Virus Res 132: 15-24, 2008. 
20. Bochkarev A, Barwell JA, Pfuetzner RA, Furey W Jr, Edwards AM and Frappier L: Crystal structure of the DNA-binding domain of the Epstein-Barr virus origin-binding protein EBNA 1. Cell 83: 39-46, 1995.

21. Ceccarelli DF and Frappier L: Functional analyses of the EBNA1 origin DNA binding protein of Epstein-Barr virus. J Virol 74: 4939-4948, 2000.

22. Wu H, Kapoor P and Frappier L: Separation of the DNA replication, segregation, and transcriptional activation functions of Epstein-Barr nuclear antigen 1. J Virol 76: 2480-2490, 2002

23. Kanda T, Otter M and Wahl GM: Coupling of mitotic chromosome tethering and replication competence in Epstein-Barr virus-based plasmids. Mol Cell Biol 21: 3576-3588, 2001.

24. Wysokenski DA and Yates JL: Multiple EBNA1-binding sites are required to form an EBNA1-dependent enhancer and to activate a minimal replicative origin within oriP of Epstein-Barr virus. J Virol 63: 2657-2666, 1989.

25. Schaefer BC, Strominger JL and Speck SH: Hostcell-determined methylation of specific Epstein-Barr virus promoters regulates the choice between distinct viral latency programs. Mol Cell Biol 17: 364-377, 1997.
26. Altmann M, Pich D, Ruiss R, Wang J, Sugden B and Hammerschmidt W: Transcriptional activation by EBV nuclear antigen 1 is essential for the expression of EBV's transforming genes. Proc Natl Acad Sci USA 103: 14188-14193, 2006.

27. Srinivas SK and Sixbey JW: Epstein-Barr virus induction of recombinase-activating genes RAG1 and RAG2. J Virol 69: 8155-8158, 1995.

28. Kube D, Vockerodt M, Weber O, Hell K, Wolf J, Haier B, Grässer FA, Müller-Lantzsch N, Kieff E, Diehl V and Tesch H: Expression of Epstein-Barr virus nuclear antigen 1 is associated with enhanced expression of CD25 in the Hodgkin cell line L428. J Virol 73: 1630-1636, 1999.

29. Kuhn-Hallek I, Sage DR, Stein L, Groelle H and Fingeroth JD: Expression of recombination activating genes (RAG-1 and RAG-2) in Epstein-Barr virus-bearing B cells. Blood 85: 1289-1299, 1995.

30. Wood VH, O'Neil JD, Wei W, Stewart SE, Dawson CW and Young LS: Epstein-Barr virus-encoded EBNA1 regulates cellular gene transcription and modulates the STAT1 and TGFbeta signaling pathways. Oncogene 26: 4135-4147, 2007. 